\title{
Would Worldwide Vaccination of Both Males and Females Against the Human Papillomavirus be a Worthy Investment?
}

\author{
Kush Patel*
}

The human papillomavirus (HPV) is the most common sexually transmitted virus, carrying a lifetime risk for women of $75 \%$. This DNA virus causes lesions of the skin and of mucous membranes (1). HPV infection is known to predispose patients to cancers of the penis, anus, vagina, vulva, cervical, oesophagus, skin and oral pharynx, accounting for $5 \%$ of all global cancer incidences. Of these, cervical cancer is of grave importance as it makes up two-thirds of all HPVassociated cancers and is a leading cause of death from cancer in women worldwide (2-4). Fortunately, pharmaceuticals have designed a bivalent (targeting HPV subtypes 16 and 18 which account for $70 \%$ of all cervical cancer cases) and a quadrivalent vaccine (targeting HPV subtypes 16, 18, 6 and 11). They have an excellent safety profile, are highly immunogenic and confer type-specific protection against HPV infection $(3,5)$. Considering the epidemiological information presented above, it may seem appropriate to introduce a worldwide vaccination programme targeting both males and females. However, there are several issues that require attention before such a program is started.

Firstly, some argue that limited financial reserves should not be used to prevent a problem that is not a national health burden. For example, cervical cancer accounts for only $0.6 \%$ of cancer deaths per year in the USA. By not implementing a vaccination program, the money saved could be diverted elsewhere, perhaps to improve the availability of pap smear testing for more women (6). In the UK, the cost of such a vaccination program would add $£ 72$ million (130 million CAD) to the current cervical cancer control program. Future studies need to elucidate whether or not this additional

*To whom correspondence should be addressed: Kush Patel

509 balmoral apartments,

2 Praed street,

London,

W2 1AL cost will lead to benefits from lower cervical cancer rates while reducing costs of screening and treatment. If this is not the case, then the vaccination program may not be an efficient use of resources (7). However this does not seem likely as one study modelling an HPV vaccination program showed that if all adolescent girls in the USA were vaccinated against high-risk HPV infection, a life expectancy gain of 2.8 days would be achieved. 1340 deaths attributed to cervical cancer, 3317 cases of cervical cancer and 112,710 cases of squamous intraepithelial lesions (a pre-malignant condition that leads to cervical cancer) could be prevented in the study population's lifetime (8). Another study showed that vaccinating females with the HPV 16/18 vaccine would reduce cervical cancer cases by $61.8 \%$ as compared with not vaccinating them (9). Conversely, if a vaccination program is not implemented, there would be the high costs of cancer treatment, follow-up appointments and physical and emotional factors to be dealt with (6).

Secondly, there is the issue over which sex should be vaccinated. Both males and females are carriers of the virus and are susceptible to the infection. Therefore, both sexes should ideally be vaccinated. Vaccinating males and females will confer herd immunity and further reduce cervical cancer cases by $2.2 \%$ compared to female-only vaccination. Men who are carriers of HPV are susceptible to oral, skin, oesophageal, anal, penile, head and neck cancers $(2,10)$, and the rates of HPV infection are higher in men who have sex with men, compared to the general population (9). While these facts may argue for vaccinating both males and females, vaccinating both sexes is not as cost-effective as vaccinating females only ( $\$ 442,039$ versus $\$ 14,583$ quality associated life years (9). Taking these statistics into account and the fact that HPV-associated disease is more common and more dangerous in women than in men, women should be given priority over men for receiving the vaccine, especially in a limited-resource 
environment. It is believed that a female-only vaccination program with a high coverage is likely to also protect males (who have sex with female partners) against HPV via herd immunity. On the other hand, in underdeveloped countries, if vaccine coverage is low, vaccinating both males and females may be more effective in preventing HPV-related cervical disease (10). Further studies are required to evaluate the effectiveness of vaccinating males before they are considered in such vaccination programs (7).

Thirdly, $80 \%$ of cervical cancer cases are found in the developing world (3) where there is a lack of adequate screening programs (10). Unfortunately, high costs associated with the vaccine and running a vaccination program make it difficult to implement the recommended 3-dose vaccination protocol in developing countries. However, by using fewer doses (two) and targeting high-risk groups, compliance would improve, costs would decrease and efficacy would still be adequate $(2,9)$. A reduction in the cost of the vaccine would make the implementation of a vaccination program in developing countries more attractive.

Fourthly, determining the age at which the vaccine should be given has been fiercely debated. Fortunately, a consensus seems to have been reached. Efficacy of the vaccine would be maximised if children were immunised before reaching sexual maturity $(3,6,9)$. According to the centre for disease control, $57 \%$ of girls between 14-19 years are sexually active (11). Additionally, the vaccines are more immunogenic in younger children than in older ones (6), but considered to be more cost-effective if given to 12 year olds rather than infants (9).

However, efforts at vaccinating children has been met with much controversy, because many parents believe that this may give children tacit consent to engage in unsafe sexual activity, even though a relation between vaccination and increased sexual activity has not been proven $(6,12)$. Indeed, culture, religion and education may impede the successful implementation of a global vaccination program. For example, it may not be possible to vaccinate 12 year olds in India because of the belief that a 'good' woman would not be promiscuous and thus does not need the vaccine (13). An important factor that needs to be addressed is adequate education about the severity and complications of HPV infection and the effectiveness of the vaccine. This may make the vaccine more acceptable to parents and children (12). In the UK, vaccine coverage of $70.6 \%$ was achieved with the first dose and $68.5 \%$ with the second dose. These rates were as expected from previous opinions but were lower than the uptake rates of meningitis $\mathrm{C}$ and hepatitis $\mathrm{B}$ vaccinations (10). Better patient education will be the key to achieving a greater coverage of the vaccine. Currently, public opinion backs the HPV vaccine so long as it is safe and effective at preventing cancer (7).

An HPV vaccine will prevent cervical cancer in the population that receive the vaccine before becoming infected. An immediate problem that our society faces today is the high prevalence of HPV infection and its associated malignant sequela. This is where effective screening programs come in. Many developed countries have the infrastructure and financial resources to run cervical cancer screening programmes using regular smear tests and colposcopies. Unfortunately this may be a huge undertaking for developing countries. Hope may come in the form of other screening tests; a recent trial carried out in rural India achieved a significant reduction in advanced cervical cancer incidence and mortality using a single round of HPV screening compared to cytology and visual inspection of the cervix with acetic acid (VIA) (14). However, before such a program is laid out, it will be important to define local prevalence rates of HPV and cervical cancer, provide a low-cost HPV test and have an infrastructure that can cater for such a program (15).

There is a definite benefit that would be gained from a global HPV vaccination program. However, advances are needed to provide a single-dose, needle-free, heatstable and affordable vaccine to overcome the socioeconomic barriers associated with cervical cancer (2). In the coming few years, as various vaccination programs unfold, results of their effectiveness and hurdles accompanying their implementation will be revealed. This will provide stronger data supporting a global vaccination programme. Many believe that even if a vaccination program is available, however, screening for cervical cancer is still essential as many women have already been infected with HPV (16). At present, there is ample evidence supporting such a program and with careful planning and adequate patient education (7), a successful and cost-effective reduction of cervical cancer is possible.

\section{REFERENCES}

1. Peter M Howley DR1, editor. Fields Virology. 5th ed; 2007.

2. Chan JK, Berek JS. Impact of the human papilloma vaccine on cervical cancer. J Clin Oncol. 2007 Jul 10;25(20):2975-82.

3. Lowy DR, Schiller JT. Prophylactic human papillomavirus vaccines. J Clin Invest. 2006 May;116(5):1167-73.

4. Howley PM. Role of the human papillomaviruses in human cancer. Cancer Res. 1991 Sep 15;51(18 Suppl):5019s-22s.

5. Stanley M. HPV vaccines. Best Pract Res Clin Obstet Gynaecol. 2006 Apr;20(2):279-93.

6. Haber G, Malow RM, Zimet GD. The HPV vaccine mandate controversy. J Pediatr Adolesc Gynecol. 2007 Dec;20(6):32531.

7. Raffle AE. Challenges of implementing human papillomavirus (HPV) vaccination policy. BMJ. 2007 Aug 25;335(7616):375-7. 
8. Sanders GD, Taira AV. Cost-effectiveness of a potential vaccine for human papillomavirus. Emerg Infect Dis. 2003 Jan;9(1):3748.

9. Taira AV, Neukermans CP, Sanders GD. Evaluating human papillomavirus vaccination programs. Emerg Infect Dis. 2004 Nov;10(11):1915-23.

10. Waller J, Wardle J. HPV vaccination in the UK. BMJ. 2008 May 10;336(7652):1028-9.

11. QuickStats: Prevalence of HPV* Infection $\uparrow$ Among Sexually Active Females Aged 14--59 Years, by Age Group --- National Health and Nutrition Examination Survey, United States, 2003-2004. 2007 [updated 2007; cited]; Available from: http://www.cdc.gov/mmwr/preview/mmwrhtml/mm5633a5.htm
12. Olshen E, Woods ER, Austin SB, Luskin M, Bauchner H. Parental acceptance of the human papillomavirus vaccine. $\mathrm{J}$ Adolesc Health. 2005 Sep;37(3):248-51.

13. Zimmerman RK. Ethical analysis of HPV vaccine policy options. Vaccine. 2006 May 29;24(22):4812-20.

14. Sankaranarayanan R, Nene BM, Shastri SS, Jayant K, Muwonge $\mathrm{R}$, Budukh AM, et al. HPV screening for cervical cancer in rural India. N Engl J Med. 2009 Apr 2;360(14):1385-94.

15. Schiffman M, Wacholder S. From India to the world--a better way to prevent cervical cancer. N Engl J Med. 2009 Apr 2;360(14):1453-5.

16. Katz IT, Wright AA. Preventing cervical cancer in the developing world. N Engl J Med. 2006 Mar 16;354(11):1110.

Kush Patel (Bsc) is a fifth year medical student at Imperial college London. He achieved a Bsc in gastroenterology and hepatology. He will be finishing medical school with a MBBS in June 2010. 